\title{
Asymptotic Upper and Lower Estimates of a Class of Positive Solutions of a Discrete Linear Equation with a Single Delay
}

\author{
J. Diblík ${ }^{1,2}$ and I. Hlavičková ${ }^{2}$ \\ ${ }^{1}$ Department of Mathematics and Descriptive Geometry, Faculty of Civil Engineering, \\ Brno University of Technology, 60200 Brno, Czech Republic \\ ${ }^{2}$ Department of Mathematics, Faculty of Electrical Engineering and Communication, \\ Brno University of Technology, 61600 Brno, Czech Republic
}

Correspondence should be addressed to J. Diblík, diblik@feec.vutbr.cz

Received 20 February 2012; Accepted 1 March 2012

Academic Editor: Elena Braverman

Copyright (C) 2012 J. Diblík and I. Hlavičková. This is an open access article distributed under the Creative Commons Attribution License, which permits unrestricted use, distribution, and reproduction in any medium, provided the original work is properly cited.

We study a frequently investigated class of linear difference equations $\Delta v(n)=-p(n) v(n-k)$ with a positive coefficient $p(n)$ and a single delay $k$. Recently, it was proved that if the function $p(n)$ is bounded above by a certain function, then there exists a positive vanishing solution of the considered equation, and the upper bound was found. Here we improve this result by finding even the lower bound for the positive solution, supposing the function $p(n)$ is bounded above and below by certain functions.

\section{Introduction}

Throughout this paper, we use the following notation: for an integer $q$, we define

$$
\mathbb{Z}_{q}^{\infty}:=\{q, q+1, \ldots\}
$$

We investigate the asymptotic behavior as $n \rightarrow \infty$ of the solutions of the discrete delayed equation of the $(k+1)$-th order

$$
\Delta v(n)=-p(n) v(n-k),
$$


where $n$ is the independent variable assuming values from the set $\mathbb{Z}_{a}^{\infty}$ with a fixed $a \in \mathbb{N}=$ $\{0,1,2, \ldots\}$. The number $k \in \mathbb{N}, k \geq 1$ is the fixed delay, $\Delta v(n)=v(n+1)-v(n)$, and $p: \mathbb{Z}_{a}^{\infty} \rightarrow$ $\mathbb{R}^{+}=(0, \infty)$.

Along with (1.2), we consider $k+1$ initial conditions

$$
v(a+s-k)=v^{a+s-k} \in \mathbb{R}, \quad s=0,1, \ldots, k .
$$

Initial problem (1.2), (1.3) obviously has a unique solution, defined for every $n \in \mathbb{Z}_{a-k}^{\infty}$. Moreover, the solution of (1.2) continuously depends on initial conditions (1.3).

Equation (1.2) is investigated very frequently. It was analyzed, for example, in papers [1-3] (where the comparison method [4,5] was used) and [6]. Similar problems for differential and dynamic equations are studied, for example, in [7-10].

In a recent work of the authors [6], it is proved that if the function $p(n)$ is bounded above by a certain function, then there exists a positive vanishing (i.e., tending to 0 as $n \rightarrow \infty$ ) solution of the considered equation. Moreover, its upper bound was found. Our aim is to improve this result and to show that if the coefficient $p(n)$ is between two functions $p_{\ell}(n)-$ $\psi(n)$ and $p_{\ell}(n)+\omega(n)$ (see (2.3), (2.6), and (2.7) below) then (1.2) has a positive vanishing solution which is bounded from below by the function $\alpha_{\ell}(n)$ (see (2.5)) and from above by the function $v_{\ell}(n)$ (see (2.4)). Due to the linearity of equation considered it becomes clear that a similar result holds for a one-parametric family of positive vanishing solutions of (1.2).

To prove this, we will use Theorem 1.1 which is one of the main results of [6]. This theorem is valid for any delayed difference equation of the form:

$$
\Delta v(n)=f(n, v(n), v(n-1), \ldots, v(n-k)) .
$$

Theorem 1.1. Let $b(n), c(n), b(n)<c(n)$ be real functions defined on $\mathbb{Z}_{a-k}^{\infty}$. Further, let $f: \mathbb{Z}_{a}^{\infty} \times$ $\mathbb{R}^{k+1} \rightarrow \mathbb{R}$ be a continuous function and let the inequalities:

$$
\begin{aligned}
& b(n)+f\left(n, b(n), v_{2}, \ldots, v_{k+1}\right)<b(n+1), \\
& c(n)+f\left(n, c(n), v_{2}, \ldots, v_{k+1}\right)>c(n+1),
\end{aligned}
$$

hold for every $n \in \mathbb{Z}_{a}^{\infty}$ and every $v_{2}, \ldots, v_{k+1}$ such that

$$
b(n-i+1)<v_{i}<c(n-i+1), \quad i=2, \ldots, k+1 .
$$

Then there exists a solution $v=v^{*}(n)$ of (1.4) satisfying the inequalities

$$
b(n)<v^{*}(n)<c(n)
$$

for every $n \in \mathbb{Z}_{a-k}^{\infty}$.

For related comparison theorems for solutions of difference equations as well as related methods and their applications, see, for example, $[1,11-21]$ and the related references therein. Investigation of positive solutions (and connected problems of oscillating solutions) attracted recently large attention. Except the references given above, one refers as well to 
[11, 22-33], and to the references therein. Existence of positive solutions of some classes of difference equations has been also studied in papers [12-16]. The existence of unbounded solutions by some comparison methods can be found, for example, in $[17,18]$.

\section{Auxiliary Functions and Lemmas}

Define the expression $\ln _{q} n, q \in \mathbb{N} \backslash\{0\}$, as

$$
\ln _{q} n:=\ln \left(\ln _{q-1} n\right),
$$

where $\ln _{0} n:=n$. We will write only $\ln n$ instead of $\ln _{1} n$. Further, for a fixed integer $\ell \geq 0$ define auxiliary functions:

$$
\begin{gathered}
\mu_{\ell}(n):=\frac{1}{8 n^{2}}+\frac{1}{8(n \ln n)^{2}}+\cdots+\frac{1}{8\left(n \ln n \cdots \ln _{\ell} n\right)^{2}} \\
p_{\ell}(n):=\left(\frac{k}{k+1}\right)^{k} \cdot\left(\frac{1}{k+1}+k \mu_{\ell}(n)\right), \\
v_{\ell}(n):=\left(\frac{k}{k+1}\right)^{n} \cdot \sqrt{n \ln n \ln _{2} n \cdots \ln _{\ell} n} \\
\alpha_{\ell}(n):=\left(\frac{k}{k+1}\right)^{n} \cdot \sqrt{n \ln n \ln _{2} n \cdots \ln _{\ell} n \ln _{\ell+1}^{-\sigma} n}
\end{gathered}
$$

where $\sigma \in \mathbb{R}, \sigma>0$, is a constant. Notice that if $a$ is sufficiently large, all these functions are well defined for $n \in \mathbb{Z}_{a}^{\infty}$.

Finally, let functions $\psi, \omega: \mathbb{Z}_{a}^{\infty} \rightarrow \mathbb{R}^{+}$satisfy for $n \in \mathbb{Z}_{a}^{\infty}$ the inequalities:

$$
\begin{gathered}
\psi(n) \leq\left(\frac{k}{k+1}\right)^{k} \cdot \frac{\delta}{\left(n \ln n \cdots \ln _{\ell} n\right)^{2} \ln _{\ell+1}^{\beta} n} \\
\omega(n) \leq \varepsilon\left(\frac{k}{k+1}\right)^{k} \cdot \frac{k(2 k-1)}{16 n^{3}}
\end{gathered}
$$

for fixed $\delta>0, \beta>2$ and $\varepsilon \in(0,1)$.

In [3], it was proved that if $p(n)$ in (1.2) is a positive function bounded above by $p_{\ell}(n)$ for some $\ell \geq 0$, then there exists a positive solution of (1.2) bounded above by the function $v_{\ell}(n)$ for $n$ sufficiently large. Since $\lim _{n \rightarrow \infty} v_{\ell}(n)=0$, such solution will vanish as $n \rightarrow \infty$. This result was further improved in [6], where it was shown that (1.2) has a positive solution bounded above by $v_{\ell}(n)$ even if the coefficient $p(n)$ satisfies a less restrictive inequality, namely, $p(n)<p_{\ell}(n)+\omega(n)$. Here we will prove that function $\alpha_{\ell}$ provides the lower estimate of the solution, supposing $p_{\ell}(n)-\psi(n) \leq p(n) \leq p_{\ell}(n)+\omega(n)$. The proof of this statement will be based on the following four lemmas. The symbols " $o$ " and " $O$ " stand for the Landau order symbols and are used as $n \rightarrow \infty$. 
Lemma 2.1. For fixed $r \in \mathbb{R} \backslash\{0\}$ and fixed $q \in \mathbb{N}$, the asymptotic representation:

$$
\begin{aligned}
\ln _{q}(n-r)= & \ln _{q} n-\frac{r}{n \ln n \cdots \ln _{q-1} n}-\frac{r^{2}}{2 n^{2} \ln n \cdots \ln _{q-1} n} \\
& -\frac{r^{2}}{2(n \ln n)^{2} \ln _{2} n \cdots \ln _{q-1} n}-\cdots-\frac{r^{2}}{2\left(n \ln n \cdots \ln _{q-1} n\right)^{2}}+o\left(\frac{1}{n^{3}}\right)
\end{aligned}
$$

holds as $n \rightarrow \infty$.

Proof. Relation (2.8) can be proved by induction with respect to $q$, for details, see [6].

Lemma 2.2. For fixed $r, s \in \mathbb{R} \backslash\{0\}$ and fixed $q \in \mathbb{N}$, the asymptotic representations:

$$
\begin{aligned}
\left(\frac{\ln _{q}(n-r)}{\ln _{q} n}\right)^{s}= & 1-\frac{r s}{n \ln n \cdots \ln _{q} n}-\frac{r^{2} s}{2 n^{2} \ln n \cdots \ln _{q} n}-\frac{r^{2} s}{2(n \ln n)^{2} \ln _{2} n \cdots \ln _{q} n}-\cdots \\
& -\frac{r^{2} s}{2\left(n \ln n \cdots \ln _{q-1} n\right)^{2} \ln _{q} n}+\frac{r^{2} s(s-1)}{2\left(n \ln n \cdots \ln _{q} n\right)^{2}}+o\left(\frac{1}{n^{3}}\right) \\
\sqrt{\frac{n-r}{n}}= & 1-\frac{r}{2 n}-\frac{r^{2}}{8 n^{2}}-\frac{r^{3}}{16 n^{3}}+o\left(\frac{1}{n^{3}}\right)
\end{aligned}
$$

hold as $n \rightarrow \infty$.

Proof. Both these relations are simple consequences of the asymptotic formula:

$$
(1-x)^{s}=1-s x+\frac{s(s-1)}{2} x^{2}-\frac{s(s-1)(s-2)}{6} x^{3}+o\left(x^{3}\right) \quad \text { as } x \longrightarrow 0 .
$$

and of Lemma 2.1 (for formula (2.9)).

In the case of relation (2.10), we put $x=r / n$ and $s=1 / 2$.

To prove relation (2.9), first notice that dividing (2.8) by $\ln _{q} n$, we get

$$
\begin{aligned}
\frac{\ln _{q}(n-r)}{\ln _{q} n}= & 1-\frac{r}{n \ln n \cdots \ln _{q-1} n \ln _{q} n}-\frac{r^{2}}{2 n^{2} \ln n \cdots \ln _{q-1} n \ln _{q} n} \\
& -\frac{r^{2}}{2(n \ln n)^{2} \ln _{2} n \cdots \ln _{q-1} n \ln _{q} n}-\cdots-\frac{r^{2}}{2\left(n \ln n \cdots \ln _{q-1} n\right)^{2} \ln _{q} n}+o\left(\frac{1}{n^{3}}\right)
\end{aligned}
$$


Thus, putting

$$
\begin{aligned}
x & =1-\frac{\ln _{q}(n-r)}{\ln _{q} n} \\
& =\frac{r}{n \ln n \cdots \ln _{q} n}+\frac{r^{2}}{2 n^{2} \ln n \cdots \ln _{q} n}+\cdots+\frac{r^{2}}{2\left(n \ln n \cdots \ln _{q-1} n\right)^{2} \ln _{q} n}+o\left(\frac{1}{n^{3}}\right)
\end{aligned}
$$

and using (2.11), we get (2.9).

The following lemma is proved in [6].

Lemma 2.3. For fixed $r \in \mathbb{R} \backslash\{0\}$ and fixed $q \in \mathbb{N}$, the asymptotic representation:

$$
\begin{aligned}
& \sqrt{\frac{(n-r)}{n} \cdot \frac{\ln (n-r)}{\ln n} \cdots \frac{\ln _{q}(n-r)}{\ln _{q} n}} \\
& \quad=1-r\left(\frac{1}{2 n}+\frac{1}{2 n \ln n}+\cdots+\frac{1}{2 n \ln n \cdots \ln _{q} n}\right)-r^{2} \mu_{q}(n)-\frac{r^{3}}{16 n^{3}}+o\left(\frac{1}{n^{3}}\right)
\end{aligned}
$$

holds as $n \rightarrow \infty$.

Lemma 2.4. For fixed $r \in \mathbb{R} \backslash\{0\}, \sigma \in \mathbb{R}^{+}$and $q \in \mathbb{N}$, the asymptotic representation:

$$
\begin{aligned}
& \sqrt{\frac{(n-r)}{n} \cdot \frac{\ln (n-r)}{\ln n} \cdots \frac{\ln _{q}(n-r)}{\ln _{q} n} \cdot \frac{\ln _{q+1}^{-\sigma}(n-r)}{\ln _{q+1}^{-\sigma} n}} \\
& =1-r\left(\frac{1}{2 n}+\frac{1}{2 n \ln n}+\cdots+\frac{1}{2 n \ln n \cdots \ln _{q} n}-\frac{\sigma}{2 n \ln n \cdots \ln _{q} n \ln _{q+1} n}\right) \\
& \quad-r^{2} \mu_{q}(n)+\frac{\sigma(\sigma+2)}{8} \cdot \frac{r^{2}}{\left(n \ln n \cdots \ln _{q+1} n\right)^{2}}-\frac{r^{3}}{16 n^{3}}+o\left(\frac{1}{n^{3}}\right)
\end{aligned}
$$

holds as $n \rightarrow \infty$.

Proof. Using Lemma 2.2 with $s=-\sigma / 2$ and $q+1$ instead of $q$, we get for $n \rightarrow \infty$

$$
\begin{aligned}
\sqrt{\frac{\ln _{q+1}^{-\sigma}(n-r)}{\ln _{q+1}^{-\sigma} n}=} & 1+\frac{r \sigma}{2 n \ln n \cdots \ln _{q+1} n}+\frac{r^{2} \sigma}{4 n^{2} \ln n \cdots \ln _{q+1} n}+\frac{r^{2} \sigma}{4(n \ln n)^{2} \ln _{2} n \cdots \ln _{q+1} n}+\cdots \\
& +\frac{r^{2} \sigma}{4\left(n \ln n \cdots \ln _{q} n\right)^{2} \ln _{q+1} n}+\frac{r^{2} \sigma(\sigma+2)}{8\left(n \ln n \cdots \ln _{q+1} n\right)^{2}}+o\left(\frac{1}{n^{3}}\right)
\end{aligned}
$$

Multiplying the asymptotic representations (2.14) and (2.16), we get (2.15). 


\section{Main Result}

Now we are ready to prove that there exists a positive solution of (1.2) which is bounded below and above. Remind the functions $p_{\ell}, v_{\ell}, \alpha_{\ell}, \psi$, and $\omega$ were defined by (2.3)-(2.6) and (2.7), respectively.

Theorem 3.1. Suppose that there exist numbers $a, \ell \in \mathbb{N}$, and $\sigma>0$, such that the function $p$ in (1.2) satisfies the inequalities

$$
0<p_{\ell}(n)-\psi(n) \leq p(n) \leq p_{\ell}(n)+\omega(n),
$$

for every $n \in \mathbb{Z}_{a}^{\infty}$. Then there exists a solution $v=v^{*}(n), n \in \mathbb{Z}_{a-k}^{\infty}$ of (1.2) such that for $n$ sufficiently large the inequalities:

$$
\alpha_{\ell}(n)<v^{*}(n)<v_{\ell}(n)
$$

hold.

Proof. Show that all the assumptions of Theorem 1.1 are fulfilled. For (1.2),

$$
f\left(n, v_{1}, \ldots, v_{k+1}\right)=-p(n) v_{k+1} .
$$

This is a continuous function. Put

$$
b(n):=\alpha_{\ell}(n), \quad c(n):=v_{\ell}(n) .
$$

We have to prove that for every $v_{2}, \ldots, v_{k+1}$ such that

$$
b(n-i+1)<v_{i}<c(n-i+1), \quad i=2, \ldots, k+1,
$$

the inequalities (1.5) and (1.6) hold for $n$ sufficiently large. Start with (1.5). That gives that for

$$
b(n-k)<v_{k+1}<c(n-k),
$$

it has to be

$$
\alpha_{\ell}(n)-p(n) \cdot v_{k+1}<\alpha_{\ell}(n+1),
$$

which is equivalent to the inequality

$$
-p(n) v_{k+1}<\alpha_{\ell}(n+1)-\alpha_{\ell}(n) .
$$


Denote the left-hand side of (3.8) as $L_{(3.8)}$. As $v_{k+1}>b(n-k)=\alpha_{\ell}(n-k)$ and as by (2.3), (2.6), and (3.1)

$$
p(n) \geq\left(\frac{k}{k+1}\right)^{k} \cdot\left(\frac{1}{k+1}+k \mu_{\ell}(n)\right)-\left(\frac{k}{k+1}\right)^{k} \cdot \frac{\delta}{\left(n \ln n \cdots \ln _{\ell} n\right)^{2} \ln _{\ell+1}^{\beta} n}
$$

we have

$$
\begin{aligned}
L_{(3.8)<}< & -\left(\frac{k}{k+1}\right)^{k}\left(\frac{1}{k+1}+k \mu_{\ell}(n)-\frac{\delta}{\left(n \ln n \cdots \ln _{\ell} n\right)^{2} \ln _{\ell+1}^{\beta} n}\right) \\
& \times\left(\frac{k}{k+1}\right)^{n-k} \sqrt{(n-k) \ln (n-k) \cdots \ln _{\ell}(n-k) \ln _{\ell+1}^{-\sigma}(n-k)} \\
= & -\left(\frac{k}{k+1}\right)^{n}\left(\frac{1}{k+1}+k \mu_{\ell}(n)-\frac{\delta}{\left(n \ln n \cdots \ln _{\ell} n\right)^{2} \ln _{\ell+1}^{\beta} n}\right) \\
& \times \sqrt{(n-k) \ln (n-k) \cdots \ln _{\ell}(n-k) \ln _{\ell+1}^{-\sigma}(n-k)} .
\end{aligned}
$$

Further, we can easily see that

$$
\begin{aligned}
& \alpha_{\ell}(n+1)-\alpha_{\ell}(n)=\left(\frac{k}{k+1}\right)^{n} \sqrt{n \ln n \cdots \ln _{\ell} n \ln _{\ell+1}^{-\sigma} n} \\
& \times\left(\frac{k}{k+1} \sqrt{\frac{(n+1)}{n} \frac{\ln (n+1)}{\ln n} \cdots \frac{\ln _{\ell}(n+1)}{\ln _{\ell} n} \frac{\ln _{\ell+1}^{-\sigma}(n+1)}{\ln _{\ell+1}^{-\sigma} n}}-1\right) \text {. }
\end{aligned}
$$

Thus, to prove (3.8), it suffices to show that for $n$ sufficiently large, the following inequality holds:

$$
\begin{aligned}
& -\left(\frac{1}{k+1}+k \mu_{\ell}(n)-\frac{\delta}{\left(n \ln n \cdots \ln _{\ell} n\right)^{2} \ln _{\ell+1}^{\beta} n}\right) \sqrt{\frac{(n-k)}{n} \frac{\ln (n-k)}{\ln n} \cdots \frac{\ln _{\ell}(n-k)}{\ln _{\ell} n} \frac{\ln _{\ell+1}^{-\sigma}(n-k)}{\ln _{\ell+1}^{-\sigma} n}} \\
& <\frac{k}{k+1} \sqrt{\frac{(n+1)}{n} \frac{\ln (n+1)}{\ln n} \cdots \frac{\ln _{\ell}(n+1)}{\ln _{\ell} n} \frac{\ln _{\ell+1}^{-\sigma}(n+1)}{\ln _{\ell+1}^{-\sigma} n}-1}
\end{aligned}
$$

Denote the left-hand side of inequality (3.12) as $L_{(3.12)}$ and the right-hand side as $R_{(3.12)}$. In the following computation we will use the fact that $\beta>2$ and

$$
\mu_{\ell}(n)=\frac{1}{8 n^{2}}+O\left(\frac{1}{n^{2} \ln ^{2} n}\right)
$$


and we will omit all the terms which are of order $o\left(1 / n^{3}\right)$. Applying Lemma 2.4 with $r=k$ and $q=\ell$, we can write

$$
\begin{aligned}
L_{(3.12)=} & -\left(\frac{1}{k+1}+k \mu_{\ell}(n)-\frac{\delta}{\left(n \ln n \cdots \ln _{\ell} n\right)^{2} \ln _{\ell+1}^{\beta} n}\right) \\
& \times\left(1-k\left(\frac{1}{2 n}+\frac{1}{2 n \ln n}+\cdots+\frac{1}{2 n \ln n \cdots \ln \ell}-\frac{\sigma}{2 n \ln n \cdots \ln _{\ell} n \ln _{\ell+1} n}\right)\right. \\
& \left.-k^{2} \mu_{\ell}(n)+\frac{\sigma(\sigma+2)}{8} \cdot \frac{k^{2}}{\left(n \ln n \cdots \ln \ln _{\ell+1} n\right)^{2}}-\frac{k^{3}}{16 n^{3}}+o\left(\frac{1}{n^{3}}\right)\right) \\
= & -\frac{1}{k+1}+\frac{k}{k+1}\left(\frac{1}{2 n}+\frac{1}{2 n \ln n}+\cdots+\frac{1}{2 n \ln n \cdots \ln n}-\frac{k_{\ell}}{2 n \ln n \cdots \ln _{\ell} n \ln _{\ell+1} n}\right) \\
& +\frac{k^{2}}{k+1} \mu_{\ell}(n)-\frac{\sigma(\sigma+2)}{8(k+1)} \cdot \frac{k^{2}}{\left(n \ln n \cdots \ln _{\ell+1} n\right)^{2}}+\frac{k^{3}}{16 n^{3}(k+1)} \\
& -k \mu_{\ell}(n)+\frac{k^{2}}{16 n^{3}}+\frac{\delta}{(n \ln n \cdots \ln \ell)^{2} \ln _{\ell+1}^{\beta} n}+o\left(\frac{1}{n^{3}}\right) \\
= & -\frac{1}{k+1}+\frac{k}{k+1}\left(\frac{1}{2 n}+\frac{1}{2 n \ln n}+\cdots+\frac{1}{2 n \ln n \cdots \ln _{\ell} n}-\frac{\sigma}{2 n \ln n \cdots \ln _{\ell} n \ln _{\ell+1} n}\right) \\
& -\frac{k}{k+1} \mu_{\ell}(n)-\frac{\sigma(\sigma+2)}{8(k+1)} \cdot \frac{k^{2}}{\left(n \ln n \cdots \ln \ln _{\ell+1} n\right)^{2}}+\frac{2 k^{3}+k^{2}}{16 n^{3}(k+1)} \\
& +\frac{\delta}{(n \ln n \cdots \ln \ell)^{2} \ln _{\ell+1}^{\beta} n}+o\left(\frac{1}{n^{3}}\right) .
\end{aligned}
$$

Using Lemma 2.4 with $r=-1$ and $q=\ell$, we get for $R_{(3.12)}$

$$
\begin{aligned}
R_{(3.12)}= & \frac{k}{k+1}\left(1+\frac{1}{2 n}+\frac{1}{2 n \ln n}+\cdots+\frac{1}{2 n \ln n \cdots \ln \ln _{\ell} n}-\frac{\sigma}{2 n \ln n \cdots \ln _{\ell} n \ln _{\ell+1} n}\right. \\
& \left.-\mu_{\ell}(n)+\frac{\sigma(\sigma+2)}{8} \cdot \frac{1}{\left(n \ln n \cdots \ln _{\ell+1} n\right)^{2}}+\frac{1}{16 n^{3}}+o\left(\frac{1}{n^{3}}\right)\right)-1 \\
= & \frac{-1}{k+1}+\frac{k}{k+1}\left(\frac{1}{2 n}+\frac{1}{2 n \ln n}+\cdots+\frac{1}{2 n \ln n \cdots \ln \ell}-\frac{\sigma}{2 n \ln n \cdots \ln _{\ell} n \ln \ell+1}\right) \\
& -\frac{k}{k+1} \cdot \mu_{\ell}(n)+\frac{\sigma(\sigma+2) k}{8(k+1)} \cdot \frac{1}{\left(n \ln n \cdots \ln _{\ell+1} n\right)^{2}}+\frac{k}{16 n^{3}(k+1)}+o\left(\frac{1}{n^{3}}\right) .
\end{aligned}
$$


It is easy to see that the inequality (3.12) reduces to

$$
\begin{aligned}
& -\frac{\sigma(\sigma+2) k^{2}}{8(k+1)} \cdot \frac{1}{\left(n \ln n \cdots \ln _{\ell+1} n\right)^{2}}+\frac{2 k^{3}+k^{2}}{16 n^{3}(k+1)}+\frac{\delta}{\left(n \ln n \cdots \ln _{\ell} n\right)^{2} \ln _{\ell+1}^{\beta} n}+o\left(\frac{1}{n^{3}}\right) \\
& <\frac{\sigma(\sigma+2) k}{8(k+1)} \cdot \frac{1}{\left(n \ln n \cdots \ln _{\ell+1} n\right)^{2}}+\frac{k}{16 n^{3}(k+1)}+o\left(\frac{1}{n^{3}}\right)
\end{aligned}
$$

This inequality is equivalent to

$$
-\frac{\sigma(\sigma+2) k}{8} \cdot \frac{1}{\left(n \ln n \cdots \ln _{\ell+1} n\right)^{2}}+\frac{k(2 k-1)}{16 n^{3}}+\frac{\delta}{\left(n \ln n \cdots \ln _{\ell} n\right)^{2} \ln _{\ell+1}^{\beta} n}+o\left(\frac{1}{n^{3}}\right)<0 .
$$

The last inequality holds for $n$ sufficiently large because $k \geq 1, \sigma>0, \beta>2$, and as $n \rightarrow \infty$,

$$
\frac{\delta}{\left(n \ln n \cdots \ln _{\ell} n\right)^{2} \ln _{\ell+1}^{\beta} n}, \quad \frac{1}{n^{3}}
$$

tend to zero faster than

$$
\frac{1}{\left(n \ln n \cdots \ln _{\ell+1} n\right)^{2}}
$$

does.

Thus, we have proved that inequality (1.5) holds.

Next, according to (1.6), we have to prove that

$$
v_{\ell}(n)-p(n) v_{k+1}>v_{\ell}(n+1)
$$

which is equivalent to the inequality:

$$
-p(n) v_{k+1}>v_{\ell}(n+1)-v_{\ell}(n)
$$

Denote the left-hand side of (3.21) as $L_{(3.21)}$. As $v_{k+1}<c(n-k)=v_{\ell}(n-k)$ and as by (2.3), (3.1), and (2.7)

$$
p(n) \leq\left(\frac{k}{k+1}\right)^{k} \cdot\left(\frac{1}{k+1}+k \mu_{\ell}(n)\right)+\varepsilon\left(\frac{k}{k+1}\right)^{k} \cdot \frac{k(2 k-1)}{16 n^{3}}
$$


we have

$$
\begin{aligned}
L_{(3.21)>} & -\left(\frac{k}{k+1}\right)^{k}\left(\frac{1}{k+1}+k \mu_{\ell}(n)+\varepsilon \cdot \frac{k(2 k-1)}{16 n^{3}}\right) \\
& \times\left(\frac{k}{k+1}\right)^{n-k} \sqrt{(n-k) \ln (n-k) \cdots \ln _{\ell}(n-k)} \\
= & -\left(\frac{k}{k+1}\right)^{n}\left(\frac{1}{k+1}+k \mu_{\ell}(n)+\varepsilon \cdot \frac{k(2 k-1)}{16 n^{3}}\right) \cdot \sqrt{(n-k) \ln (n-k) \cdots \ln _{\ell}(n-k)} .
\end{aligned}
$$

Further, we can easily see that

$$
v_{\ell}(n+1)-v_{\ell}(n)=\left(\frac{k}{k+1}\right)^{n} \sqrt{n \ln n \cdots \ln n}\left(\frac{k}{k+1} \sqrt{\frac{(n+1)}{n} \cdot \frac{\ln (n+1)}{\ln n} \cdots \frac{\ln _{\ell}(n+1)}{\ln _{\ell} n}}-1\right) \text {. }
$$

Thus, to prove (3.21), it suffices to show that for $n$ sufficiently large, the following inequality holds:

$$
\begin{gathered}
-\left(\frac{1}{k+1}+k \mu_{\ell}(n)+\varepsilon \cdot \frac{k(2 k-1)}{16 n^{3}}\right) \sqrt{\frac{(n-k)}{n} \cdot \frac{\ln (n-k)}{\ln n} \cdots \frac{\ln _{\ell}(n-k)}{\ln _{\ell} n}} \\
>\frac{k}{k+1} \sqrt{\frac{(n+1)}{n} \cdot \frac{\ln (n+1)}{\ln n} \cdots \frac{\ln _{\ell}(n+1)}{\ln _{\ell} n}}-1 .
\end{gathered}
$$

Denote the left-hand side of inequality (3.25) as $L_{(3.25)}$ and the right-hand side as $R_{(3.25)}$. Using Lemma 2.3 with $r=k$ and $q=\ell$, we can write

$$
\begin{aligned}
L_{(3.25)=} & -\left(\frac{1}{k+1}+k \mu_{\ell}(n)+\varepsilon \cdot \frac{k(2 k-1)}{16 n^{3}}\right) \\
& \times\left(1-k\left(\frac{1}{2 n}+\frac{1}{2 n \ln n}+\cdots+\frac{1}{2 n \ln n \cdots \ln \ell}\right)-k^{2} \mu_{\ell}(n)-\frac{k^{3}}{16 n^{3}}+o\left(\frac{1}{n^{3}}\right)\right) \\
= & -\frac{1}{k+1}+\frac{k}{k+1}\left(\frac{1}{2 n}+\frac{1}{2 n \ln n}+\cdots+\frac{1}{2 n \ln n \cdots \ln n}\right) \\
& +\frac{k^{2}}{k+1} \mu_{\ell}(n)+\frac{k^{3}}{16 n^{3}(k+1)}-k \mu_{\ell}(n)+\frac{k^{2}}{16 n^{3}}-\varepsilon \cdot \frac{k(2 k-1)}{16 n^{3}}+o\left(\frac{1}{n^{3}}\right) \\
= & -\frac{1}{k+1}+\frac{k}{k+1}\left(\frac{1}{2 n}+\frac{1}{2 n \ln n}+\cdots+\frac{1}{2 n \ln n \cdots \ln n}\right) \\
& -\frac{k}{k+1} \mu_{\ell}(n)+\frac{k^{3}}{16 n^{3}(k+1)}+\frac{k(k-\varepsilon(2 k-1))}{16 n^{3}}+o\left(\frac{1}{n^{3}}\right) .
\end{aligned}
$$


Using Lemma 2.3 with $r=-1$ and $q=\ell$, we get for $R_{(3.25)}$

$$
\begin{aligned}
R_{(3.25)}= & \frac{k}{k+1}\left(1+\frac{1}{2 n}+\frac{1}{2 n \ln n}+\cdots+\frac{1}{2 n \ln n \cdots \ln n}-\mu_{\ell}(n)+\frac{1}{16 n^{3}}+o\left(\frac{1}{n^{3}}\right)\right)-1 \\
= & \frac{-1}{k+1}+\frac{k}{k+1}\left(\frac{1}{2 n}+\frac{1}{2 n \ln n}+\cdots+\frac{1}{2 n \ln n \cdots \ln n}\right) \\
& -\frac{k}{k+1} \cdot \mu_{\ell}(n)+\frac{k}{16 n^{3}(k+1)}+o\left(\frac{1}{n^{3}}\right)
\end{aligned}
$$

It is easy to see that the inequality (3.25) reduces to

$$
\frac{k^{3}}{16 n^{3}(k+1)}+\frac{k(k-\varepsilon(2 k-1))}{16 n^{3}}+o\left(\frac{1}{n^{3}}\right)>\frac{k}{16 n^{3}(k+1)}+o\left(\frac{1}{n^{3}}\right)
$$

This inequality is equivalent to

$$
\frac{k(2 k-1)(1-\varepsilon)}{16 n^{3}}+o\left(\frac{1}{n^{3}}\right)>0
$$

The last inequality holds for $n$ sufficiently large because $k \geq 1$ and $1-\varepsilon \in(0,1)$. We have proved that all the assumptions of Theorem 1.1 are fulfilled and hence there exists a solution of (1.2) satisfying conditions (1.8), that is, in our case, conditions (3.2).

\section{Acknowledgments}

The research was supported by the Grant P201/10/1032 of the Czech Grant Agency (Prague), by the Council of Czech Government Grant MSM 0021630519 and by the Grant FEKT-S-11-2921 of Faculty of Electrical Engineering and Communication, Brno University of Technology.

\section{References}

[1] J. Baštinec and J. Diblík, "Subdominant positive solutions of the discrete equation $\Delta u(k+n)=$ - p(k)u(k)," Abstract and Applied Analysis, no. 6, pp. 461-470, 2004.

[2] J. Bašstinec and J. Diblík, "Remark on positive solutions of discrete equation $\Delta u(k+n)=-p(k) u(k)$, , Nonlinear Analysis, vol. 63, pp. e2145-e2151, 2005.

[3] J. Baštinec, J. Diblík, and Z. Šmarda, "Existence of positive solutions of discrete linear equations with a single delay," Journal of Difference Equations and Applications, vol. 16, no. 9, pp. 1047-1056, 2010.

[4] I. Györi and G. Ladas, Oscillation Theory of Delay Differential Equations, The Clarendon Press Oxford University Press, New York, NY, USA, 1991.

[5] G. Ladas, C. G. Philos, and Y. G. Sficas, "Sharp conditions for the oscillation of delay difference equations," Journal of Applied Mathematics and Simulation, vol. 2, no. 2, pp. 101-111, 1989.

[6] J. Diblík and I. Hlavičková, "Asymptotic behavior of solutions of delayed difference equations," Abstract and Applied Analysis, vol. 2011, Article ID 671967, 24 pages, 2011.

[7] L. Berezansky, J. Diblík, and Z. Šmarda, “On connection between second-order delay differential equations and integro-differential equations with delay," Advances in Difference Equations, vol. 2010, Article ID 143298, 8 pages, 2010.

[8] E. Braverman and B. Karpuz, "Nonoscillation of first-order dynamic equations with several delays," Advances in Difference Equations, vol. 2010, Article ID 873459, 22 pages, 2010. 
[9] E. Braverman and B. Karpuz, "Nonoscillation of second-order dynamic equations with several delays," Abstract and Applied Analysis, vol. 2011, Article ID 591254, 34 pages, 2011.

[10] J. Diblík and N. Koksch, "Positive solutions of the equation $i(t)=-c(t) x(t-\tau)$ in the critical case," Journal of Mathematical Analysis and Applications, vol. 250, no. 2, pp. 635-659, 2000.

[11] R. P. Agarwal, M. Bohner, and W.-T. Li, Nonoscillation and Oscillation: Theory for Functional Differential Equations, Marcel Dekker, New York, NY, USA, 2004.

[12] L. Berg and S. Stević, "On the asymptotics of the difference equation $y_{n}\left(1+y_{n-1} \ldots y_{n-k+1}\right)=y_{n-k}$," Journal of Difference Equations and Applications, vol. 17, no. 4, pp. 577-586, 2011.

[13] S. Stević, "Asymptotic behavior of a class of nonlinear difference equations," Discrete Dynamics in Nature and Society, vol. 2006, Article ID 47156, 10 pages, 2006.

[14] S. Stević, "On positive solutions of a $(k+1)$-th order difference equation," Applied Mathematics Letters, vol. 19, no. 5, pp. 427-431, 2006.

[15] S. Stević, "Existence of nontrivial solutions of a rational difference equation," Applied Mathematics Letters, vol. 20, no. 1, pp. 28-31, 2007.

[16] S. Stevich, "Nontrivial solutions of higher-order rational difference equations," Mathematical Notes, vol. 84, no. 5-6, pp. 718-724, 2008.

[17] S. Stević, "On the recursive sequence $x_{n+1}=\max \left\{c, x_{n}^{p} / x_{n-1}^{p}\right\}, "$ Applied Mathematics Letters, vol. 21, no. 8, pp. 791-796, 2008.

[18] S. Stević, "On a nonlinear generalized max-type difference equation," Journal of Mathematical Analysis and Applications, vol. 376, no. 1, pp. 317-328, 2011.

[19] S. Stević, "On a system of difference equations," Applied Mathematics and Computation, vol. 218, no. 7, pp. 3372-3378, 2011.

[20] S. Stević, "On some solvable systems of difference equations," Applied Mathematics and Computation, vol. 218, no. 9, pp. 5010-5018, 2012.

[21] S. Stević, "On the difference equation $x_{n}=x_{n-2} /\left(b_{n}+c_{n} x_{n-1} x_{n-2}\right), "$ Applied Mathematics and Computation, vol. 218, pp. 4507-4513, 2011.

[22] J. Baštinec, L. Berezansky, J. Diblík, and Z. Šmarda, “On the critical case in oscillation for differential equations with a single delay and with several delays," Abstract and Applied Analysis, vol. 2010, Article ID 417869, 20 pages, 2010.

[23] J. Baštinec, J. Diblík, and B. Zhang, "Existence of bounded solutions of discrete delayed equations," in Proceedings of the Sixth International Conference on Difference Equations, pp. 359-366, CRC, Boca Raton, Fla, usa, 2004.

[24] L. Berezansky and E. Braverman, "On existence of positive solutions for linear difference equations with several delays," Advances in Dynamical Systems and Applications, vol. 1, no. 1, pp. 29-47, 2006.

[25] G. E. Chatzarakis, C. G. Philos, and I. P. Stavroulakis, "An oscillation criterion for linear difference equations with general delay argument," Portugaliae Mathematica, vol. 66, no. 4, pp. 513-533, 2009.

[26] G. E. Chatzarakis, R. Koplatadze, and I. P. Stavroulakis, "Oscillation criteria of first order linear difference equations with delay argument," Nonlinear Analysis, vol. 68, no. 4, pp. 994-1005, 2008.

[27] J. Čermák, "Asymptotic bounds for linear difference systems," Advances in Difference Equations, vol. 2010, Article ID 182696, 14 pages, 2010.

[28] I. Gyóri and M. Pituk, "Asymptotic formulae for the solutions of a linear delay difference equation," Journal of Mathematical Analysis and Applications, vol. 195, no. 2, pp. 376-392, 1995.

[29] T. Khokhlova, M. Kipnis, and V. Malygina, "The stability cone for a delay differential matrix equation," Applied Mathematics Letters, vol. 24, no. 5, pp. 742-745, 2011.

[30] L. K. Kikina and I. P. Stavroulakis, "Oscillation criteria for second-order delay, difference, and functional equations," International Journal of Differential Equations, vol. 2010, Article ID 598068, 14 pages, 2010.

[31] B. Krasznai, I. Győri, and M. Pituk, "Positive decreasing solutions of higher-order nonlinear difference equations," Advances in Difference Equations, vol. 2010, Article ID 973432, 16 pages, 2010.

[32] R. Medina and M. Pituk, "Nonoscillatory solutions of a second-order difference equation of Poincaré type," Applied Mathematics Letters, vol. 22, no. 5, pp. 679-683, 2009.

[33] A. Zafer, "The existence of positive solutions and oscillation of solutions of higher-order difference equations with forcing terms," Computers $\mathcal{E}$ Mathematics with Applications, vol. 36, no. 10-12, pp. 27-35, 1998. 


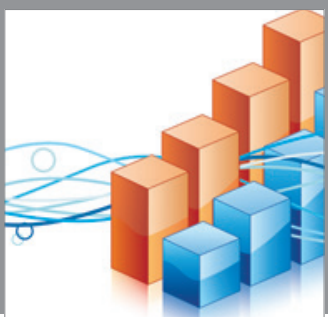

Advances in

Operations Research

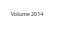

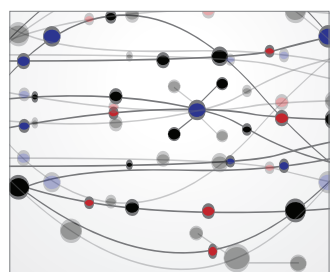

\section{The Scientific} World Journal
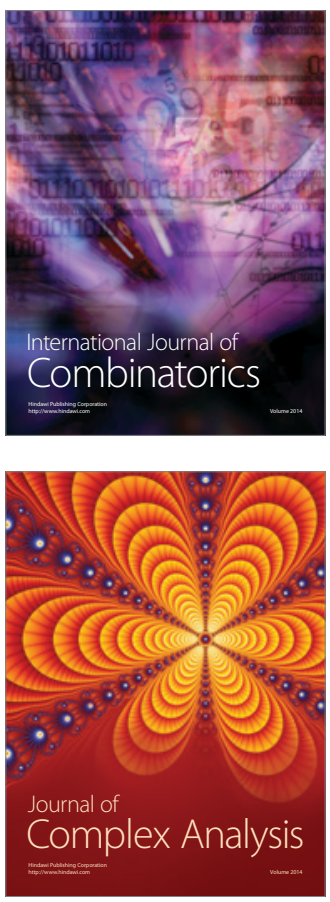

International Journal of

Mathematics and

Mathematical

Sciences
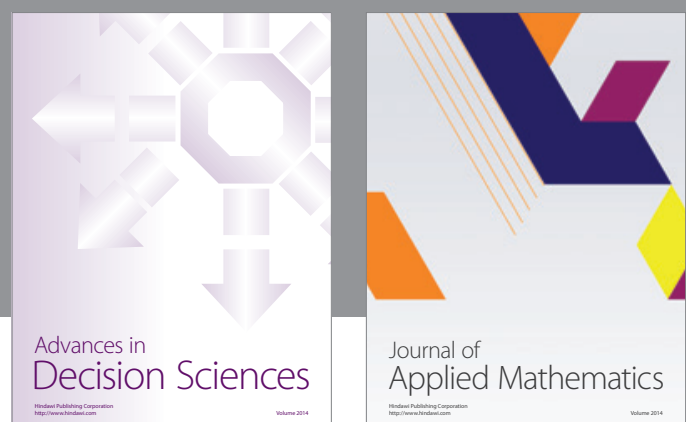

Journal of

Applied Mathematics
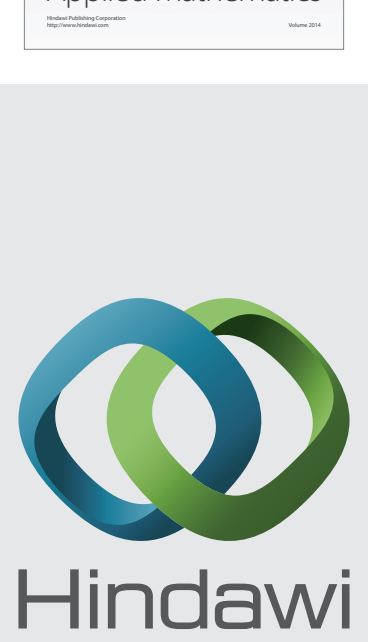

Submit your manuscripts at http://www.hindawi.com
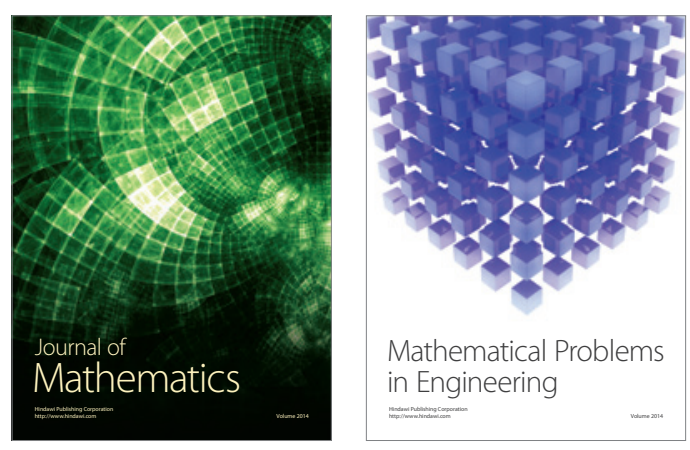

Mathematical Problems in Engineering
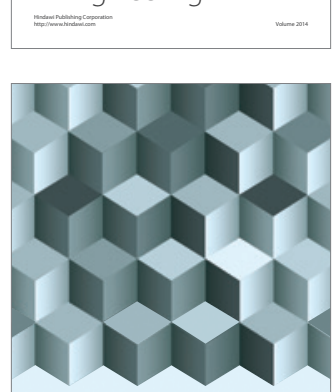

Journal of

Function Spaces
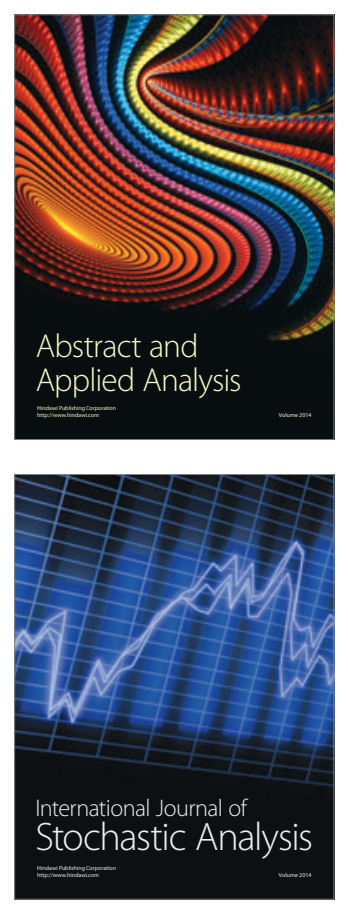

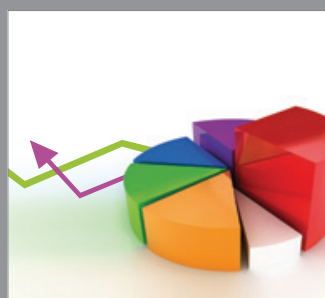

ournal of

Probability and Statistics

Promensencen
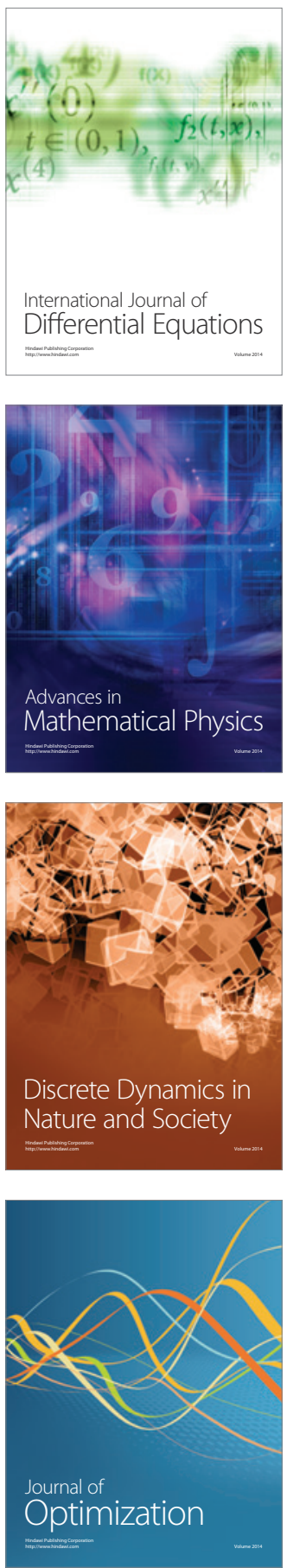